\title{
Finite element formulation and algorithms for unsaturated soils. Part I: Theory
}

\author{
Daichao Sheng ${ }^{1, *, \dagger}$, Scott W. Sloan ${ }^{1}$, Antonio Gens ${ }^{2}$ and David W. Smith ${ }^{1}$ \\ ${ }^{1}$ Discipline of Civil, Surveying \& Environmental Engineering, The University of Newcastle, NSW 2308, Australia \\ ${ }^{2}$ Civil Engineering School, Technical University of Catalonia (UPC), Barcelona, Spain
}

\begin{abstract}
SUMMARY
This paper presents a complete finite-element treatment for unsaturated soil problems. A new formulation of general constitutive equations for unsaturated soils is first presented. In the incremental stress-strain equations, the suction or the pore water pressure is treated as a strain variable instead of a stress variable. The global governing equations are derived in terms of displacement and pore water pressure. The discretized governing equations are then solved using an adaptive time-stepping scheme which automatically adjusts the time-step size so that the integration error in the displacements and pore pressures lies close to a specified tolerance. The non-linearity caused by suction-dependent plastic yielding, suction-dependent degree of saturation, and saturation-dependent permeability is treated in a similar way to the elastoplasticity. An explicit stress integration scheme is used to solve the constitutive stress-strain equations at the Gauss point level. The elastoplastic stiffness matrix in the Euler solution is evaluated using the suction as well as the stresses and hardening parameters at the start of the subincrement, while the elastoplastic matrix in the modified Euler solution is evaluated using the suction at the end of the subincrement. In addition, when applying subincrementation, the same rate is applied to all strain components including the suction. Copyright (C) 2003 John Wiley \& Sons, Ltd.
\end{abstract}

KEY WORDS: unsaturated soils; finite element method; constitutive model; stress integration; time integration

\section{INTRODUCTION}

Current methods for deformation analysis of unsaturated soil problems use either an uncoupled hydrological approach or a fully coupled hydro-mechanical approach. In the former, the continuity equations for fluid flow are first solved by assuming a non-deformable body [1,2]. The information so obtained is then used to solve the mechanical equilibrium equations for the displacements if required. One disadvantage of this approach is the inconsistency between the continuity equations for a non-deformable body and the mechanical equilibrium equations for a deformable body. Although attractive because of its simplicity, this procedure is clearly limited to soils that do not experience significant volume changes over time.

\footnotetext{
*Correspondence to: D. Sheng, Discipline of Civil, Surveying and Environmental Engineering, The University of Newcastle, NSW 2308, Australia.

${ }^{\dagger}$ E-mail: daichao.sheng@newcastle.edu.au

Copyright (C) 2003 John Wiley \& Sons, Ltd.

Received August 2001

Revised August 2002

and March 2003
} 
The coupled hydro-mechanical approach, on the other hand, is more rigorous as it is based on the continuity equations for deformable media (see, for example [3-5]). These equations are coupled with the mechanical equilibrium relations through the volumetric strain rate and the relationships between the stresses and pore pressures, and are solved simultaneously. Compared to the uncoupled hydrological approach, this type of formulation is conceptually more complete and has become the mainstream in deformation analysis for unsaturated soils.

Mathematical equations for unsaturated soil problems can be derived from either physical or thermodynamic principles, and a number of different formulations have been presented in the literature [3-8]. These all contain at least three sets of partial differential equations; one for mechanical equilibrium, one for pore water flow, and the other for pore air flow. Some formulations also consider other processes like heat transfer and transport of chemical components. While these aspects are undoubtedly important for certain engineering problems, they will not be considered here.

When discretized by the finite-element method, the governing equations for an unsaturated soil lead to a system of first-order differential equations. Due to the elastoplastic nature of the soil skeleton, and the suction dependency of the degree of saturation and the permeability, all coefficient matrices in the these equations are non-linear. This is clearly different from the standard consolidation formulation for a saturated soil, where only the elastoplastic stiffness matrix is non-linear. The governing equations for an unsaturated soil are also distinguished from those for a saturated soil in that they are usually asymmetric and ill conditioned. Due to

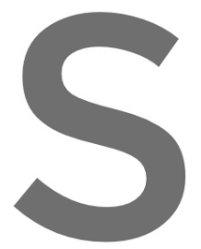
the relatively recent behaviour, very litt models in practical implicit scheme which problems [9]. Gatmit of these procedures develo
researcl
inite-ele
h was f
et al. [8
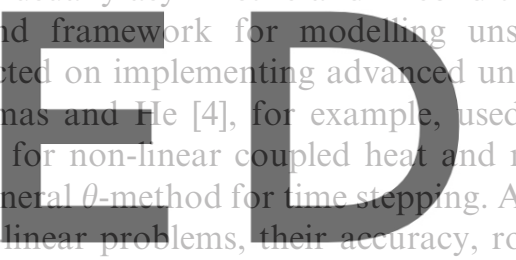
satur
mois
Altho
obus urated sol
turated s
mid-poil
isture flc
hough bo efficiency are far from certain. The ubiquitous midpoint (Crank-Nicholson) scheme, for changes in the forcing function, and often requires special smoothing procedures [10] Moreover, all of these standard methods require the user to select the time steps by hand. This often leads to inaccurate or inefficient solutions, as the optimum time-step strategy for these complex problems is impossible to guess in advance.

An important element in a coupled hydro-mechanical problem is the mechanical constitutive model adopted for the soil. During the past decade a great number of constitutive models for unsaturated soil have been developed, mostly based on experimental observations and plasticity theory. The well-known model of Alonso et al. [11] uses two independent stress variables, the total stress in excess of pore air pressure and the suction (the pore air pressure in excess of pore water pressure), as a basis for its formulation. A loading-collapse yield surface defines the variation of the yield surface with the suction and the modified Cam clay model [12] is adopted for the fully saturated state. Similar constitutive models to that of Alonso et al. [11] include those of Schrefler and Zhan [13], Cui et al. [14], Bolzon et al. [15], Wheeler and Sivakumar [16], Kohgo et al. [17], and Modaressi and Abou Bekr [18]. A thorough review of these can be found in Gens [19]. More recently, Loret and Khalili [5] proposed a constitutive model based on an effective stress concept. They argue that the effective stress, if defined properly, provides a compact and rigorous description of unsaturated soil behaviour. This argument has been examined experimentally by Geiser [20] and Khalili [21], who showed that a single effective 
stress is capable of predicting the shear strength and volume change of unsaturated soils accurately. However, in their formulation of the constitutive model, Loret and Khalili [5] include the suction as an independent variable in the yield function and plastic potential, in addition to the effective stresses and the suction-dependent hardening parameter. This makes their model similar to those of Alonso et al. [11], which use two stress variables.

In order to implement an unsaturated soil model in a finite-element code, the stresses are updated by integrating the stress-strain relations with a known strain increment. The methods available for doing this can be classified broadly into two categories: implicit or explicit. Implicit methods, which solve the non-linear constitutive equations by iteration, are generally accurate but are difficult to implement since they require second derivatives of the plastic potential to be evaluated at a range of stress states. Moreover, they are not especially robust and require special procedures to be used when the iteration process fails to converge. Explicit methods, on the other hand, are generally robust but, depending on their method of implementation, can be very inaccurate In their simplest form, these procedures use the gradients of the yield surface and plastic potential at the start of the strain increment, and their accuracy can only be controlled by breaking up the strain increment into subincrements (see, for example, $[22,23]$ ). The relative performance of implicit and explicit methods is strongly dependent on the precise form of the constitutive model. While stress integration methods for saturated constitutive models have been well studied, research on numerical algorithms for evaluating unsaturated soil constitutive

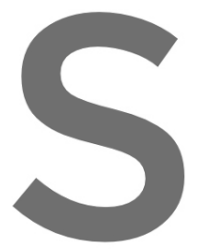
relations is very limited. The donstitutive relations for the latter the former as they and different harden the suction is treated equations in the san difficulties in traditi volve different stress sta
as a stress variable, even 1
way as the displacement
nal stress integration sch many constituti
tholgh it can be
or strains. This
emes. Recently, are quite distinct from
rent plastic yjelding b
e models for unsatura
btained direct y from the comp
eature can cause coint. [24] pres those for
ted soils,
he global
utational implicit stress integration scheme based upon the closet-point projection method [25]. This uses

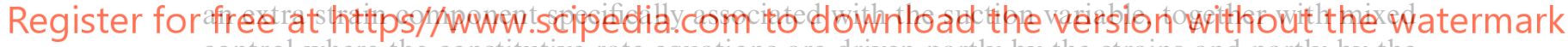
control where the constitutive rate equations are driven partly by the strains and partly by the stresses. Due to the introduction of the extra component, the strains in the constitutive equations are no longer directly related to the displacements, and have to be transferred to total strains. Although the scheme was validated against laboratory data, it did not converge for certain loading patterns. In a separate development, Zhang et al. [26] extended the implicit return-mapping algorithm of Simo and Taylor [27] to integrate the partially saturated constitutive model of Bolzon et al. [15]. As they ignore the suction rate in the consistency condition, this variable is missing from their stress-strain relations (which are purely a function of the stress and strain rates). This results in the stress-strain relations being similar to those for a saturated soil, except that some parameters, such as the plastic modulus, are influenced by the suction variable. Although their formulation of the constitutive equations greatly simplifies the stress integration scheme, it is not mathematically rigorous.

This paper presents an alternative finite-element formulation for geotechnical problems involving unsaturated soils. A framework for a general unsaturated constitutive model is first described, with the suction being treated as a strain variable. The global differential equations are then derived in terms of displacement and pore water pressure. To solve these equations, an explicit scheme with error control is used to integrate the purely strain-driven constitutive law at 
the stress point level, while an implicit adaptive time-stepping scheme is used to compute the field quantities at the global level.

\section{CONSTITUTIVE MODELLING}

\section{Stress-strain-pore pressure relations}

The constitutive equations are first described in.a general form and then illustrated using the modified Cam clay model of Roscoe and Burland [12] and the model of Alonso et al. [11]. As the air pressure remains constant (usually atmospheric) for many geotechnical problems, it is not treated as a variable in this paper. Thus, the suction is simply replaced by a negative pore water pressure. For problems with variable pore air pressure, the formulation can be generalized by using the true suction (pore air pressure in excess of pore water pressure).

The signs of the strains, stresses and pressures follow the convention of continuum mechanics, with positive stresses/pressures in tension and positive strains in dilation. In addition, all stresses and pressures are measured with respect to atmospheric pressure.

\section{Constitutive stresses. The constitutive stress is defined as}

$$
\sigma^{\prime}=\sigma-\mathbf{m} \varphi\left(u_{\mathrm{W}}\right) u_{\mathrm{W}}=\sigma-\mathbf{m} \varphi\left(S_{\mathrm{r}}\right) u_{\mathrm{W}}
$$

where $\sigma$ is the total stress vector, $\sigma^{\prime}$ is the constitutive stress vector, $m$ is the column vector with 1

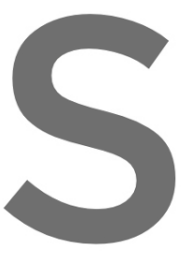

at normal stress en that depends upon The constitutive stress 2000). Since it is not stress.
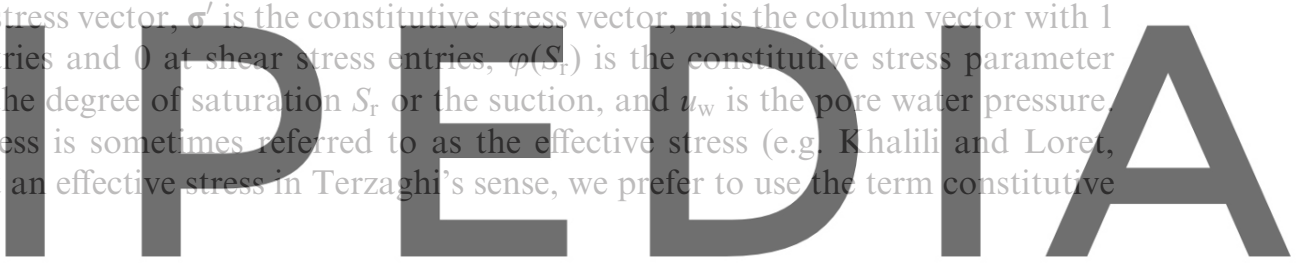

Yield function and plastic potential. The yield function $f$ and plastic potential $g$ are defined in

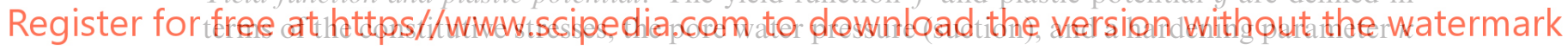

that controls the evolution of the functions according to

$$
\begin{gathered}
f\left(\boldsymbol{\sigma}^{\prime}, u_{\mathrm{W}}, \kappa\right)=0 \\
g\left(\boldsymbol{\sigma}^{\prime}, u_{\mathrm{w}} \kappa\right)=0
\end{gathered}
$$

Flow rule. The plastic strain increments are assumed normal to the plastic potential surface, so that

$$
\mathrm{d} \varepsilon^{\mathrm{p}}=\mathrm{d} \lambda \frac{\partial g}{\partial \boldsymbol{\sigma}^{\prime}}
$$

where $\mathrm{d} \lambda$ is a non-negative scalar multiplier.

Yield surface evolution. The yield surface location (the preconsolidation pressure $p_{\mathrm{c}}$ at zero suction) is taken as the hardening parameter and its evolution is given by the law

$$
\mathrm{d} \kappa=\mathrm{d} p_{\mathrm{c}}=\frac{\partial p_{\mathrm{c}}}{\partial \varepsilon_{\mathrm{v}}^{\mathrm{p}}} \mathrm{d} \varepsilon_{\mathrm{v}}^{\mathrm{p}}
$$

where $\mathrm{d} \kappa$ is the hardening parameter increment and $\varepsilon_{\mathrm{v}}^{\mathrm{p}}$ is the plastic volumetric strain. 
Consistency condition. For the stress state to remain on the yield surface, the following condition must be satisfied:

$$
\mathrm{d} f=\left(\frac{\partial f}{\partial \boldsymbol{\sigma}^{\prime}}\right)^{\mathrm{T}} \mathrm{d} \boldsymbol{\sigma}^{\prime}+\frac{\partial f}{\partial u_{\mathrm{w}}} \mathrm{d} u_{\mathrm{w}}+\frac{\partial f}{\partial \kappa} \mathrm{d} \kappa=0
$$

Note that the term $\left(\partial f / \partial u_{\mathrm{w}}\right) \mathrm{d} u_{\mathrm{w}}$ is ignored in a number of existing models for unsaturated soils (see, for example [26]). This leads to stress-strain equations which are functions of the stress and strain rates only, but is an incomplete formulation.

Stress-strain relations. The incremental stress-strain relations can be written as

$$
\mathrm{d} \boldsymbol{\sigma}^{\prime}=\mathbf{D}_{\mathrm{e}} \mathrm{d} \boldsymbol{\varepsilon}^{\mathrm{e}}=\mathbf{D}_{\mathrm{e}}\left(\mathrm{d} \boldsymbol{\varepsilon}-\mathrm{d} \boldsymbol{\varepsilon}^{\mathrm{p}}\right)
$$

where $\mathbb{D}_{\mathrm{e}}$ is the elastic stiffness matrix, $\mathrm{d}_{\varepsilon}^{\mathrm{e}}$ is the elastic strain increment, de the total strain increment, and $\mathrm{d} \varepsilon^{\mathrm{p}}$ the plastic strain increment.

Plastic multiplier d $\lambda$. Solving Equations (4)-(7) for the plastic multiplier d $\lambda$ gives

$$
\mathrm{d} \lambda=\frac{\mathbf{a}_{f}^{\mathrm{T}} \mathbf{D}_{\mathrm{e}} \mathrm{d} \varepsilon+C \mathrm{~d} u_{\mathrm{w}}}{A+\mathbf{a}_{f}^{\mathrm{T}} \mathbf{D}_{\mathrm{e}} \mathbf{a}_{g}}
$$

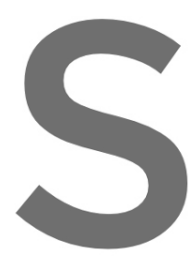

where
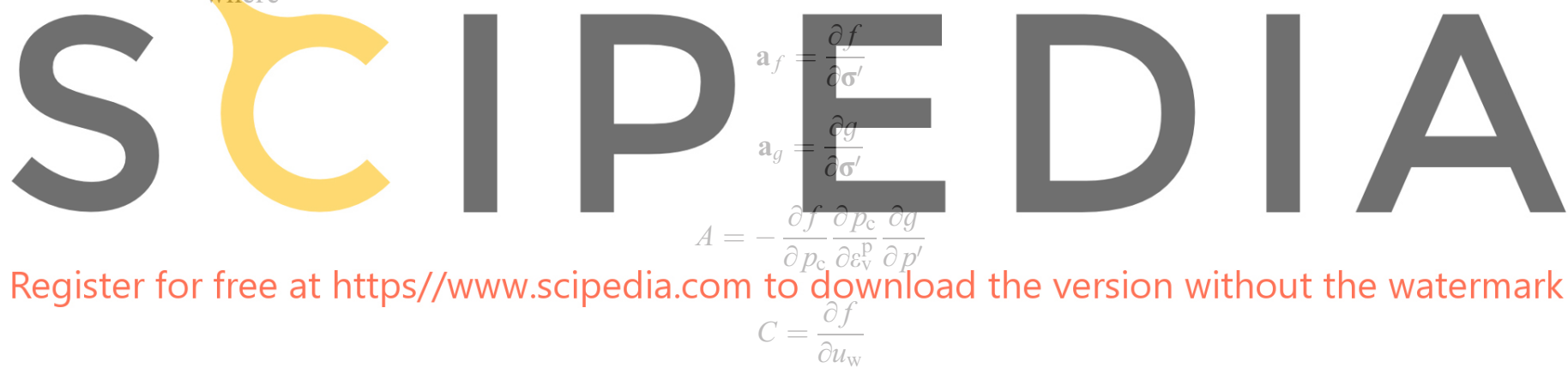

Constitutive equations. Combining Equations (4), (7) and (8) gives

$$
\begin{gathered}
\mathrm{d} \boldsymbol{\sigma}^{\prime}=\mathbf{D}_{\mathrm{ep}} \mathrm{d} \boldsymbol{\varepsilon}+\mathbf{W}_{\mathrm{ep}} \mathrm{d} u_{\mathrm{w}} \\
\mathrm{d} \kappa=\mathbf{R}_{\mathrm{ep}} \mathrm{d} \boldsymbol{\varepsilon}+Q \mathrm{~d} u_{\mathrm{w}}
\end{gathered}
$$

where

$$
\begin{gathered}
\mathbf{D}_{\mathrm{ep}}=\mathbf{D}_{\mathrm{e}}-\frac{\mathbf{D}_{\mathrm{e}} \mathbf{a}_{g} \mathbf{a}_{f}^{\mathrm{T}} \mathbf{D}_{\mathrm{e}}}{A+\mathbf{a}_{f}^{\mathrm{T}} \mathbf{D}_{\mathrm{e}} \mathbf{a}_{g}} \\
\mathbf{W}_{\mathrm{ep}}=-\frac{\mathbf{D}_{\mathrm{e}} \mathbf{a}_{g} C}{A+\mathbf{a}_{f}^{\mathrm{T}} \mathbf{D}_{\mathrm{e}} \mathbf{a}_{g}} \\
\mathbf{R}_{\mathrm{ep}}=B \frac{\mathbf{a}_{f}^{\mathrm{T}} \mathbf{D}_{\mathrm{e}}}{A+\mathbf{a}_{f}^{\mathrm{T}} \mathbf{D}_{\mathrm{e}} \mathbf{a}_{g}}
\end{gathered}
$$




$$
\begin{gathered}
Q=\frac{B C}{A+\mathbf{a}_{f}^{\mathrm{T}} \mathbf{D}_{\mathrm{e}} \mathbf{a}_{g}} \\
B=\frac{\partial p_{\mathrm{c}}}{\partial \varepsilon_{\mathrm{v}}^{\rho}} \frac{\partial g}{\partial p^{\prime}}
\end{gathered}
$$

Note that $\mathbf{W}_{\text {ep }}$ is a column vector and $\mathbf{R}_{\mathrm{ep}}$ is a row vector. Treating the pore pressure increment as an extra strain increment we obtain

$$
\Delta \boldsymbol{\varepsilon}^{\prime}=\left\{\begin{array}{c}
\Delta \boldsymbol{\varepsilon} \\
\Delta u_{\mathrm{w}}
\end{array}\right\}, \quad \mathbf{D}_{\mathrm{ep}}^{\prime}=\left\{\mathbf{D}_{\mathrm{ep}} \mathbf{W}_{\mathrm{ep}}\right\}, \quad \mathbf{R}_{\mathrm{ep}}^{\prime}=\left\{\mathbf{R}_{\mathrm{ep}} Q\right\}
$$

and the constitutive equations (9) and (10) can be rewritten as

$$
\begin{gathered}
\mathrm{d} \boldsymbol{\sigma}^{\prime}=\mathbf{D}_{\mathrm{ep}}^{\prime} \mathrm{d} \boldsymbol{\varepsilon}^{\prime} \\
\mathrm{d} \kappa=\mathbf{R}_{\mathrm{ep}}^{\prime} \mathrm{d} \boldsymbol{\varepsilon}^{\prime}
\end{gathered}
$$

The equations above are of the same overall form as those for saturated soils.
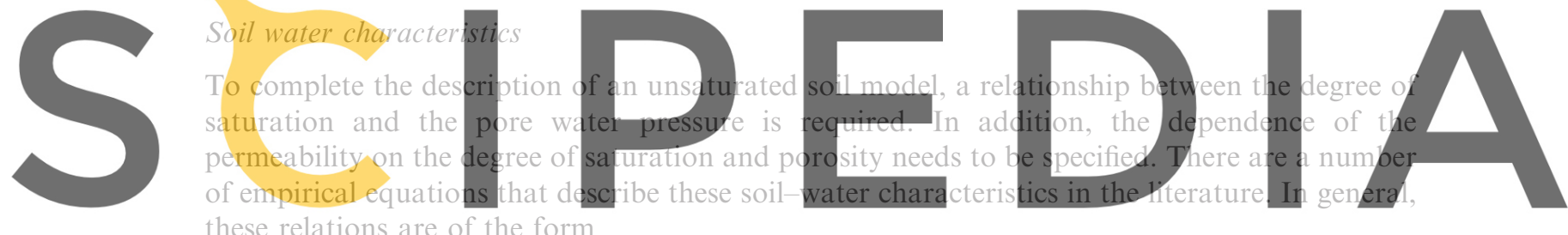

these relations are of the form

Register for free at https//www.scipedia.comprto download the version without the watermark

$$
k=k\left(S_{1}, n\right)
$$

where $S_{\mathrm{r}}$ is the degree of saturation, $k$ is the permeability, and $n$ is the porosity. Two well-known examples of the above are due to van Genuchten [28] and Hillel [29], who suggested the expressions

$$
\begin{gathered}
S_{\mathrm{r}}=\frac{1}{\left(1+\left(u_{\mathrm{w}} / a\right)^{b}\right)^{c}} \\
k=k_{\mathrm{S}} S_{\mathrm{r}}^{m}
\end{gathered}
$$

where $a, b, c$ and $m$ are constants, and $k_{\mathrm{s}}$ is the saturated permeability.

\section{GOVERNING EQUATIONS}

In its general form, the deformation of unsaturated soils involves the flow of pore fluids, mechanical equilibrium, heat transfer and possibly transport of chemical components. A comprehensive model that includes all these aspects is complex and requires a large number of 
material parameters. To make the theory more accessible to practising engineers, it is necessary to isolate the most important processes and approximate the effect of the others. Temperature, for example mainly affects the pore fluid phase change rates, except in cases where thermal stresses are significant (such as in nuclear waste disposal). This suggests that it is possible to treat the temperature as a prescribed parameter if it can be measured easily or, otherwise, solve it independently from the heat transfer equation. Similarly, it is possible to handle the pore air pressure, which is constant in many practical situations, as a prescribed function. The phase change between liquid water and vapour moisture can be effectively modelled by appropriate boundary conditions or source terms. Therefore, the simplest formulations which capture the key features of unsaturated soil behaviour will be based on mass conservation of water and mechanical equilibrium of the total soil volume. The development of such a hydromechanical model can also provide a good platform to tackle more general problems, including those with thermal and chemical effects.

\section{Mechanical equilibrium}

The equations of equilibrium can be expressed in the compact form

$$
\bar{\nabla}^{\mathrm{T}} \boldsymbol{\sigma}+\mathbf{b}=\mathbf{0}
$$

where $\bar{\nabla}$ is the differential operator and b is the body force vector. Applying the Green-Gauss

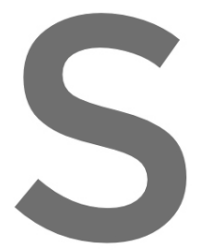
theorem and Galerkin

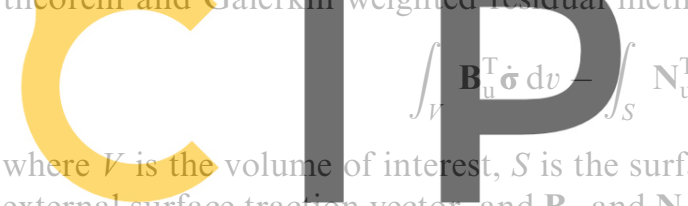

external surface traction vect

function matrices defined as

Register for free at https//www.scipedia.com $\mathrm{u}_{\mathrm{u}} \underline{\mathrm{g}} \mathrm{f}_{\mathrm{u}} \mathrm{wwnload}$ the version without the watermark

$$
\begin{aligned}
\dot{\boldsymbol{\varepsilon}} & =\mathbf{B}_{\mathrm{u}} \dot{\mathbf{U}} \\
\mathbf{B}_{\mathrm{u}} & =\bar{\nabla} \mathbf{N}_{\mathrm{u}}
\end{aligned}
$$

with $\mathbf{U}$ denoting the nodal displacement vector and the superior dot denoting a derivative with respect to time. Since

$$
\begin{gathered}
\dot{\boldsymbol{\sigma}}=\dot{\boldsymbol{\sigma}}^{\prime}+\varphi\left(S_{\mathrm{r}}\right) \mathbf{m} \dot{u}_{\mathrm{w}} \\
\dot{\boldsymbol{\sigma}}^{\prime}=\mathbf{D}_{\mathrm{ep}} \dot{\boldsymbol{\varepsilon}}+\mathbf{W}_{\mathrm{ep}} \dot{u}_{\mathrm{w}} \\
\dot{\mathbf{u}}_{\mathrm{w}}=\mathbf{N}_{\mathrm{w}} \dot{\mathbf{U}}_{\mathrm{w}}
\end{gathered}
$$

where $+\mathbf{U}_{\mathrm{w}}$ is the nodal pore pressure vector and $\mathbf{N}_{\mathrm{w}}$ is the pore pressure shape function matrix, we have

$$
\mathbf{K}_{\mathrm{ep}} \dot{\mathbf{U}}+\mathbf{L} \dot{\mathbf{U}}_{\mathrm{w}}=\dot{\mathbf{F}}^{\mathrm{ext}}
$$


with

$$
\begin{gathered}
\mathbf{K}_{\mathrm{ep}}=\sum \int \mathbf{B}_{\mathrm{u}}^{\mathrm{T}} \mathbf{D}_{\mathrm{ep}} \mathbf{B}_{\mathrm{u}} \mathrm{d} v \\
\mathbf{L}=\sum \int \mathbf{B}_{\mathrm{u}}^{\mathrm{T}} \mathbf{W}_{\mathrm{ep}} \mathbf{N}_{\mathrm{w}} \mathrm{d} v+\sum \int \mathbf{B}_{\mathrm{u}}^{\mathrm{T}} \mathbf{m} \varphi\left(S_{\mathrm{r}}\right) \mathbf{N}_{\mathrm{w}} \mathrm{d} v \\
\dot{\mathbf{F}}^{\text {ext }}=\sum \int \mathbf{N}_{\mathrm{u}}^{\mathrm{T}} \dot{\mathrm{t}} \mathrm{d} s+\sum \int \mathbf{N}_{\mathrm{u}}^{\mathrm{T}} \dot{\mathbf{b}} \mathrm{d} v
\end{gathered}
$$

The summation signs above indicate that the matrices are computed element by element and assembled in the usual manner to form the global matrices.

\section{Mass conservation for pore water}

\section{Considering the mass balance of pore fluids leads to the continuity equation of flow}

$$
\operatorname{div}\left(\rho_{\mathrm{W}} \mathbf{v}\right)+\frac{\partial}{\partial t}\left(\rho_{\mathrm{W}} n S_{\mathrm{r}}\right)=0
$$

where $\rho_{\mathrm{w}}$ is the density of pore fluid and $\mathrm{v}$ is the Darcian velocity vector. Since
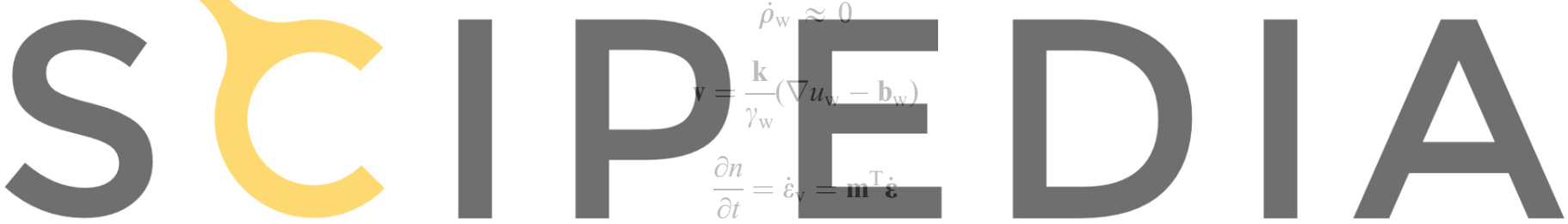

where $\mathbf{k}$ is the permeability matrix, $\gamma_{\mathrm{w}}$ is the unit weight of pore fluid, and $\mathbf{b}_{\mathrm{w}}$ is a body force

Register for ffeee

$$
\operatorname{div}\left(\frac{\mathrm{k}}{\gamma_{\mathrm{w}}}\left(\nabla u_{\mathrm{w}}-\mathrm{b}_{\mathrm{w}}\right)\right)+S_{\mathrm{r}} \mathrm{m}^{\mathrm{T}} \dot{\varepsilon}+n \frac{\partial S_{\mathrm{r}}}{\partial u_{\mathrm{w}}} \dot{u}_{\mathrm{W}}=0
$$

Discretizing $u_{\mathrm{w}}$ in space and applying the Green-Gauss theorem and Galerkin weighted residual method to Equation (15) leads to

$$
\mathbf{L}^{\prime} \dot{\mathbf{U}}+\dot{\mathbf{S U}}_{\mathrm{w}}+\dot{\mathbf{H}} \mathbf{U}_{\mathrm{w}}=\dot{\mathbf{Q}}^{\mathrm{ext}}
$$

where

$$
\begin{gathered}
\mathbf{L}^{\prime}=\sum \int \mathbf{N}_{\mathrm{w}}^{\mathrm{T}} S_{\mathrm{r}} \mathbf{m}^{\mathrm{T}} \mathbf{B}_{\mathrm{u}} \mathrm{d} v \\
\mathbf{S}=\sum \int \mathbf{N}_{\mathrm{w}}^{\mathrm{T}} n \frac{\partial S_{\mathrm{r}}}{\partial u_{\mathrm{w}}} \mathbf{N}_{\mathrm{w}} \mathrm{d} v \\
\dot{\mathbf{H}}=-\sum \int \mathbf{B}_{\mathrm{w}}^{\mathrm{T}} \frac{\mathbf{k}}{\gamma_{\mathrm{w}}} \mathbf{B}_{\mathrm{w}} \mathrm{d} v \\
\dot{\mathbf{Q}}^{\mathrm{ext}}=\sum \int \mathbf{N}_{\mathrm{w}}^{\mathrm{T}} q \mathrm{~d} s+\sum \int \mathbf{B}_{\mathrm{w}}^{\mathrm{T}} \frac{\mathbf{k}}{\gamma_{\mathrm{w}}} \mathbf{b}_{\mathrm{w}} \mathrm{d} v
\end{gathered}
$$


and $q$ is the prescribed fluid flux on the boundary of the domain. The matrix $\mathbf{H}$ and the vector $\mathbf{Q}^{\text {ext }}$ are written in rate form because the permeability and the flux are rate-type quantities.

\section{Coupled equations}

Equations (14) and (16) can be rewritten in the following matrix form:

$$
\left[\begin{array}{ll}
\mathbf{K}_{\mathrm{ep}} & \mathbf{L} \\
\mathbf{L}^{\prime} & \mathbf{S}
\end{array}\right]\left\{\begin{array}{c}
\dot{\mathbf{U}} \\
\dot{\mathbf{U}}_{\mathrm{w}}
\end{array}\right\}+\left[\begin{array}{cc}
\mathbf{0} & \mathbf{0} \\
\mathbf{0} & \dot{\mathbf{H}}
\end{array}\right]\left\{\begin{array}{c}
\mathbf{U} \\
\mathbf{U}_{\mathrm{w}}
\end{array}\right\}=\left\{\begin{array}{c}
\dot{\mathbf{F}}^{\text {ext }} \\
\dot{\mathbf{Q}}^{\text {ext }}
\end{array}\right\}
$$

This system is characterized by its non-linearity, asymmetry and ill-conditioning. The nonlinearity occurs because of the elastoplastic stiffness response $\left(\mathbf{K}_{\mathrm{ep}}\right.$ and $\left.\mathbf{L}\right)$, the suction-dependent degree of saturation $\left(\mathbf{L}, \mathbf{L}^{\prime}\right.$ and $\left.\mathbf{S}\right)$, and the suction-dependent permeability $(\dot{\mathbf{H}})$. This situation is markedly different from the standard formulation for coupled consolidation analysis of a saturated soil, where usually only the matrix $\mathbf{K}_{\mathrm{ep}}$ is non-linear. Asymmetry arises when a nonassociated flow rule is used to describe the deformation of the soil skeleton or when the soil becomes unsaturated $\left(\mathbf{L}^{T} \neq \mathbf{L}^{\prime}\right)$. A further complication with these equations is that, in general, the elements in the matrices $\mathbf{K}_{\mathrm{ep}}, \mathbf{L}, \mathbf{L}^{\prime}$ and $\mathbf{S}$ are usually not of the same order of magnitude. Indeed, the terms in these matrices may differ by a factor of $10^{6}$ or more, so that the equations frequently become very ill-conditioned.

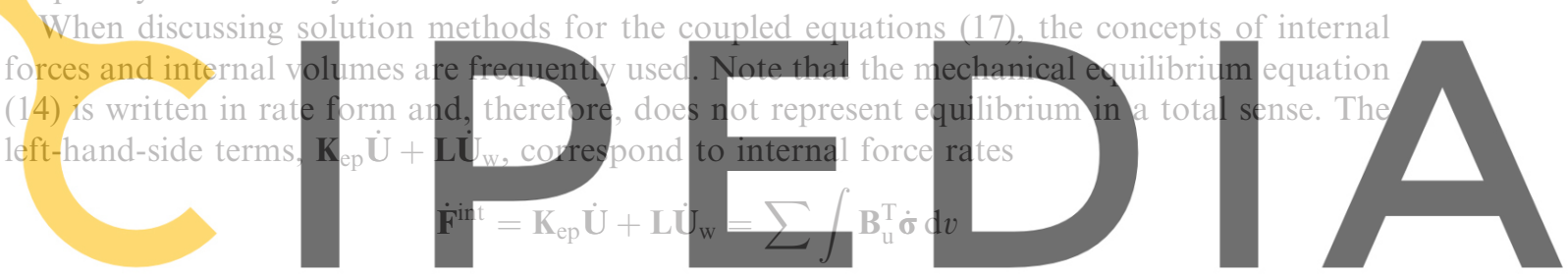

while the right-hand-side term, $\mathbb{F}^{\text {ext }}$, corresponds to the applied external force rates. Equation

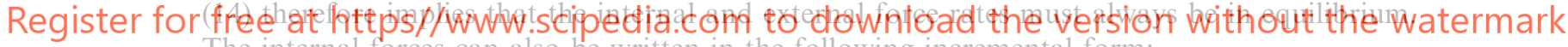

The internal forces can also be written in the following incremental form:

$$
\Delta \mathbf{F}^{\mathrm{int}}=\mathbf{K}_{\mathrm{ep}} \Delta \mathbf{U}+\mathbf{L} \Delta \mathbf{U}_{\mathrm{w}}=\sum \int \mathbf{B}_{\mathrm{u}}^{\mathrm{T}} \Delta \boldsymbol{\sigma} \mathrm{d} v
$$

where $\Delta \mathbf{F}^{\text {int }}$ are the internal force increments for the displacement increments $\Delta \mathbf{U}$ and pore pressure increments $\Delta \mathbf{U}_{\mathrm{w}}$, and $\Delta \boldsymbol{\sigma}$ are the total stress increments.

The mass balance equation (16) is written in terms of volume rates of pore fluid. As the pore fluid is assumed to be incompressible, the rate of change of fluid volume is identical to the rate of change of fluid mass. The left-hand side terms, $\mathbf{L}^{\prime} \mathbf{U}+\mathbf{S} \dot{\mathbf{U}}_{\mathrm{w}}+\dot{\mathbf{H}} \mathbf{U}_{\mathrm{w}}$, correspond to the rate of internal volume change of the pore fluid

$$
\dot{\mathbf{Q}}^{\text {int }}=\mathbf{L}^{\prime} \dot{\mathbf{U}}+\mathbf{S} \dot{\mathbf{U}}_{\mathrm{w}}+\dot{\mathbf{H}} \mathbf{U}_{\mathrm{w}}=\sum \int \mathbf{N}_{\mathrm{w}}^{\mathrm{T}} \frac{\partial\left(n S_{\mathrm{r}}\right)}{\partial t} \mathrm{~d} v+\sum \int \mathbf{B}_{\mathrm{w}}^{\mathrm{T}} \mathbf{v} \mathrm{d} v
$$

while the right-hand side, $\dot{\mathbf{Q}}^{\text {ext }}$, corresponds to the flow rate of external pore fluid addition. Therefore, Equation (16) implies that the rates of internal and external volume change of pore fluid must balance. The internal volume change can also be written in the incremental form

$$
\Delta \mathbf{Q}^{\text {int }}=\mathbf{L}^{\prime} \Delta \mathbf{U}+\mathbf{S} \Delta \mathbf{U}_{\mathrm{w}}+h \dot{\mathbf{H}} \mathbf{U}_{\mathrm{w}}=\sum \int \mathbf{N}_{\mathrm{w}}^{\mathrm{T}} \Delta\left(n S_{\mathrm{r}}\right) \mathrm{d} v+\sum \int \mathbf{B}_{\mathrm{w}}^{\mathrm{T}}(h \mathbf{v}) \mathrm{d} v
$$


where $\Delta \mathbf{Q}^{\text {int }}$ is the internal volume increment for given displacement increments $\Delta \mathbf{U}$, pore pressure increments $\Delta \mathbf{U}_{\mathrm{w}}$, and total pore pressures $\mathbf{U}_{\mathrm{w}}, h$ is the time step for $\Delta \mathbf{U}$ and $\Delta \mathbf{U}_{\mathrm{w}}$, and $\Delta\left(n S_{\mathrm{r}}\right)$ is the moisture content increment. Note that the internal volume increments are related to the pore pressure increments as well as the total pore pressures. This is different from the internal force increments.

\section{ADAPTIVE TIME-STEPPING SCHEME}

The solution of the coupled system (17) requires integration to be performed over a discrete number of time increments. Traditionally, most time-stepping schemes are based on the ubiquitous $\theta$-method. In this procedure, all time-dependent quantities in (17) are represented by a weighted sum of the values at the start and end of the time step, respectively, with the rate quantities being approximated by average rates over the time step. The stability and accuracy of the $\theta$-method depend on the weighting parameter $\theta$, and have been investigated by Booker and Small (1975) [30] and Vermeer and Verruijt (1981) [31] for soil consolidation problems. In a more general vein, Wood (1990) gives stability and accuracy results for the scheme when it is applied to any system of first-order differential equations. The $\theta$-method has been used to solve coupled systems of equations for unsaturated soil problems in a number of studies, including those of Thomas and He [4] and Gatmiri et al. [8].

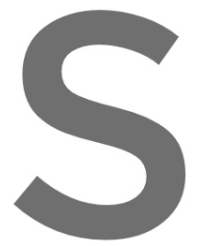

More recently, S elastoplastic consoli mesh, the temporal each time step, the between a first-orde Gladwell [35] solution. By choosing the integrationn
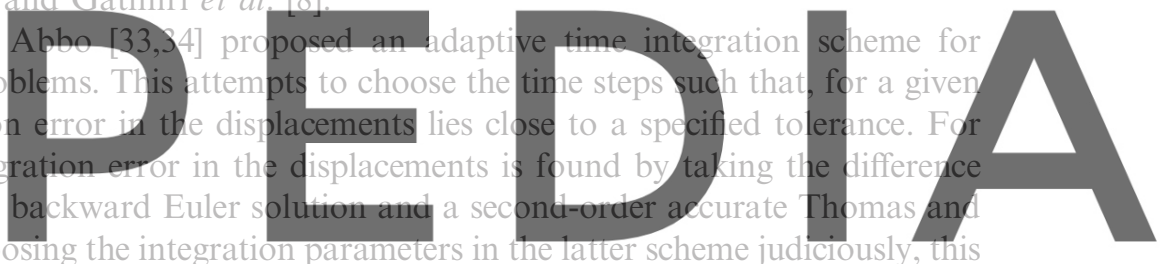
error measure can be computed at negligible additional cost. Unlike other existing solution

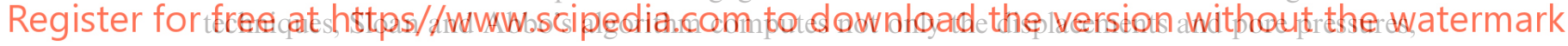

but also their derivatives with respect to time. The performance of this adaptive time-stepping scheme has been tested on a wide range of elastic and elastoplastic saturated soil problems and, in all cases, it was able to constrain the global temporal error in the displacements to lie near the desired tolerance $[33,34,36]$. In general, the behaviour of the automatic procedure is largely insensitive to the size and distribution of the initial trial time steps, and its performance compares favourably to that of the conventional backward Euler scheme. To achieve solutions of similar accuracy, the automatic and backward Euler schemes use a similar amount of computational effort. The chief advantage of the automatic method is that it removes the need to determine the time-stepping error by an empirical trial-and-error procedure.

In this section, the adaptive algorithm of Sloan and Abbo $[33,34]$ is enhanced to handle the non-linear coupled systems of equations that stem from unsaturated soil behaviour.

\section{Local solution error and adaptive time stepping}

The governing equations (17) can be written as a system of non-linear equations of the form

$$
\mathbf{R}(\mathbf{X}, \dot{\mathbf{X}})=\dot{\mathbf{W}}^{\text {ext }}-\mathbf{C}_{\mathrm{ep}}(\mathbf{X}) \dot{\mathbf{X}}-\mathbf{K}(\mathbf{X}) \mathbf{X}=\mathbf{0}
$$


where

$$
\mathbf{C}_{\mathrm{ep}}(\mathbf{X})\left[\begin{array}{ll}
\mathbf{K}_{\mathrm{ep}} & \mathbf{L} \\
\mathbf{L}^{\prime} & \mathbf{S}
\end{array}\right], \quad \mathbf{K}(\mathbf{X})=\left[\begin{array}{cc}
\mathbf{0} & \mathbf{0} \\
\mathbf{0} & \dot{\mathbf{H}}
\end{array}\right], \quad \dot{\mathbf{W}}^{\text {ext }}=\left\{\begin{array}{c}
\dot{\mathbf{F}}^{\text {ext }} \\
\dot{\mathbf{Q}}^{\text {ext }}
\end{array}\right\}
$$

and $\mathbf{X}=\left\{\mathbf{U}, \mathbf{U}_{\mathrm{w}}\right\}^{\mathrm{T}}$ with $\dot{\mathbf{X}}=\left\{\dot{\mathbf{U}}, \dot{\mathbf{U}}_{\mathrm{w}}\right\}^{\mathrm{T}}$.

Following Sloan and Abbo $[30,31]$ the local truncation error for a given time step is measured by taking the difference between a pair of first- and second-order solutions which are provided by the backward Euler and Thomas and Gladwell methods. It is assumed that a series of coarse time steps are defined initially which, if required, are subdivided into substeps to keep the local error below a specified tolerance. Both the backward Euler and Thomas and Gladwell methods are unconditionally stable and have good damping characteristics ([32]).

Applying the first-order-accurate backward Euler method to Equation (18) leads to

$$
\mathbf{C}_{\mathrm{ep}} \dot{\mathbf{X}}_{n}+\mathbf{K}\left(\mathbf{X}_{n-1}+h \dot{\mathbf{X}}_{n}\right)=\dot{\mathbf{W}}_{n}^{\text {ext }}
$$

while the second-order-accurate Thomas and Gladwell method gives

$$
\mathbf{C}_{\mathrm{ep}}\left(\dot{\mathbf{X}}_{n-1}+h \mathbf{A}\right)+\mathbf{K}\left(\mathbf{X}_{n-1}+h \dot{\mathbf{X}}_{n-1}+h^{2} \mathbf{A}\right)=\dot{\mathbf{W}}_{n}^{\mathrm{ext}}
$$

With the same starting values $\mathbf{X}_{n-1}$ these two schemes lead to an identical system of equations if

$$
\dot{\mathbf{X}}_{n}=\dot{\mathbf{X}}_{n-1}+h \mathbf{A}
$$

Solving (19) for $\dot{\mathbf{X}}_{n}$, the first-order-accurate update is

$$
\tilde{\mathbf{X}}_{n}=\mathbf{X}_{n-1}+h \dot{\mathbf{X}}_{n}
$$

while the second-order-accurate update is obtained as

$$
\begin{aligned}
& \mathbf{A}=\frac{\dot{\mathbf{X}}_{n}-\dot{\mathbf{X}}_{n-1}}{h} \\
& \mathbf{X}_{n}=\mathbf{X}_{n-1}+h \dot{\mathbf{X}}_{n-1}+\frac{1}{2} h^{2} \mathbf{A}=\mathbf{X}_{n-1}+\frac{h}{2} \dot{\mathbf{X}}_{n}+\frac{h}{2} \dot{\mathbf{X}}_{n-1}
\end{aligned}
$$

with $\dot{\mathbf{X}}_{n-1}$ assumed to be known.

The local error estimate is given by the difference between the first-order and second-orderaccurate solutions according to

$$
\mathbf{E}_{n}=h \dot{\mathbf{X}}_{n-1}+\frac{1}{2} h^{2} \mathbf{A}-h \dot{\mathbf{X}}=\frac{h}{2}\left(\dot{\mathbf{X}}_{n-1}-\dot{\mathbf{X}}_{n}\right)
$$

For the purpose of error control, $\mathbf{E}_{n}$ may be replaced by the dimensionless relative error measure

$$
R_{n}=\max \left\{\frac{\left\|\mathbf{E}_{n}^{u}\right\|}{\left\|\mathbf{U}_{n}\right\|}, \frac{\left\|\mathbf{E}_{n}^{\mathrm{p}}\right\|}{\left\|\mathbf{P}_{n}\right\|}\right\}
$$

where $(\mathbf{U}, \mathbf{P})$ are the displacement and pore pressure components of $\mathbf{X}$ and $\left(\mathbf{E}_{n}^{u}, \mathbf{E}_{n}^{\mathrm{p}}\right)$ are the local errors in (U, $\mathbf{P})$. The current time subincrement is accepted if $R_{n}$ is less than some specified tolerance on the local integration error, DTOL, and rejected otherwise. In either case, the size of the next time step $h_{n+1}$, is found from

$$
h_{n+1}=q h_{n}
$$


where $q$ is a factor which is chosen to limit the predicted truncation error (see References $[33,34])$.

Once the velocity $\dot{\mathbf{X}}_{n}$ is found, the relative error $R_{n}$ is calculated using Equation (23) and compared with the prescribed tolerance DTOL. If the subincrement is accepted, the unknown $\mathbf{X}_{n}$ can be computed using either the first-order-accurate update (21) or the second-orderaccurate update (22). The latter option, which was used by Sloan and Abbo [33,34], creates a lag between the unbalanced forces and the displacements, as the unbalanced forces are checked only at the end of the first-order-accurate update. This error caused by this lag is usually small, and can be neglected for simple models such as Mohr-Coulomb. For more complicated models, however, such as those belonging to the critical state family, the unbalanced forces after the second-order update should be checked and additional Network-Raphson iterations performed if necessary. In this paper, the first-order-accurate update is used to propagate the analysis.

\section{EXPLICIT STRESS INTEGRATION}

One special feature of the constitutive equations for unsaturated soils is the dependence of the yield surface on the suction variable. Many models, such as the one proposed by Alonso et al . [11], treat the suction as an additional stress variable and add an extra strain variable to match it [24]. The constitutive equations in this case are not purely driven by strain but, rather, are of a mixed form with six known strain increments and one known stress increment. This causes problems in stress integration, as the suction is a stress variable in the yield function/plastic potential and the final stress state must lie on the known suction plane. If an implicit stress integration scheme is used, convergence problems are expected once an elastic trial stress path crosses a non-convex yield surface from outside. For unsaturated soils, the so-called load collapse yield surface in the suction versus mean stress plane is not guaranteed to be convex. Some non-convex load-collapse yield surfaces will be shown in Part II that follows. On the other hand, if an explicit scheme is used, then the strain subincrementation may have to be carried out at a different rate to the suction (stress) subincrementation for sufficient accuracy to be obtained. This causes difficulties in the integration process, as it requires two separate integration steps to be performed.

In the model described here, the suction is treated as an additional strain component, even though it affects the yield function in a similar manner to the two stress-variable models. The advantage of this approach is that the constitutive equations are then similar to those for saturated soils and are purely strain driven. This leads to a formulation which is consistent with the conventional displacement finite-element method, where the displacements/pore pressures are found first and then the strains and then the stresses. The constitutive relations, Equations (11) and (12), can be solved by a wide range of implicit and explicit methods, but here we will focus on the explicit scheme with automatic subincrementation described by Sloan [23] and Sloan et al. [37]. This method is attractive for finite-element applications because it attempts to control the errors in the stresses and hardening parameter arising from the approximate integration of the constitutive law.

Given a set of strain increments, an initial stress state and an initial yield surface, the explicit scheme finds the final stress state and the final yield surface according to the following steps: 
1. Elastic trial stress: Assume the given strain increment $\Delta \boldsymbol{\varepsilon}^{\prime}$ is purely elastic and compute a trial stress state $\boldsymbol{\sigma}_{\mathrm{e}}^{\prime}$. Determine if the trial stress state is outside the current yield surface, i.e. if it causes plastic yielding. If the trial stress state is inside or on the current yield surface, so that no plastic yielding occurs, update the stress state with the trial stress state and exit.

2. Intersection with yield surface: If the trial stress state is found to cause plastic yielding, find the intersection point $\boldsymbol{\sigma}_{\text {int }}^{\prime}$ between the trial stress path and the current yield surface. This is characterized by the portion of the strain increment, $\alpha$, that causes plastic yielding. Update the stress state with the intersection point $\boldsymbol{\sigma}_{\text {int }}^{\prime}$ and the strain increment with $\alpha \Delta \varepsilon^{\prime}$.

3. Stress integration: Integrate the elastoplastic stress-strain equations over the strain increment and find the final stress state and the new yield surface location. If necessary, subincrement the strain increment.

4. Yield surface drift correction: If the final stress state lies outside the updated yield surface, project the stresses back to the yield surface using a drift correction method.

Step 4 closely follows that in Reference [37] and hence will not be repeated here. Steps 1-3 also follow those in Reference [37] in principle. However, because the suction or the pore water pressure is physically a stress variable (it plays a similar role to the stresses in the yield function and plastic potential), but can conveniently be treated as a strain variable, evaluation of suctiondependent variables during stress integration is not straightforward. Therefore, we will provide all necessary details for Steps 1-3 here, even though this means some overlap with the paper by Sloan et al. [37].

\section{Elastic trial stress state and yield surface intersection}

Given a vector of imposed strain increments $\Delta \boldsymbol{\varepsilon}^{\prime}$, an elastic trial stress increment $\Delta \boldsymbol{\sigma}_{\mathrm{e}}^{\prime}$ can be found according to

$$
\Delta \boldsymbol{\sigma}_{\mathrm{e}}^{\prime}=\mathbf{D}_{\mathrm{e}}^{\prime} \Delta \boldsymbol{\varepsilon}^{\prime}
$$

In the modified Cam clay (MCC) model adopted here, $\Delta \boldsymbol{\sigma}_{\mathrm{e}}^{\prime}$ does not depend on the suction increment and, hence, $\Delta \boldsymbol{\varepsilon}^{\prime}$ can be replaced by $\Delta \boldsymbol{\varepsilon}$ and $\mathbf{D}_{\mathrm{e}}^{\prime}$ by $\mathbf{D}_{\mathrm{e}}$. However, to keep the formulation general, we will use the extended strain increment $\Delta \boldsymbol{\varepsilon}^{\prime}$ and the extended stiffness matrix $\mathbf{D}_{\mathrm{e}}^{\prime}$. If the elastic part of the constitutive relation is linear, the stress-strain matrix $\mathbf{D}_{\mathrm{e}}^{\prime}$ is independent of the stresses and it is trivial to compute the elastic trial state $\boldsymbol{\sigma}_{\mathrm{e}}^{\prime}=\boldsymbol{\sigma}_{0}^{\prime}+\Delta \boldsymbol{\sigma}_{\mathrm{e}}^{\prime}$ (where $\boldsymbol{\sigma}_{0}^{\prime}$ is the initial stress state). For non-linear elastic behaviour, however, the above equation must be integrated to compute the proper value of $\Delta \boldsymbol{\sigma}_{\mathrm{e}}^{\prime}$. In MCC elasticity, the incremental relation between the mean stress and the elastic volumetric strain can be integrated analytically to give the secant elastic moduli [33]. This permits the corresponding secant elastic stiffness matrix $\overline{\mathbf{D}}_{\mathrm{e}}^{\prime}$, to be formed and relation (24) is replaced by

$$
\Delta \boldsymbol{\sigma}_{\mathrm{e}}^{\prime}=\overline{\mathbf{D}}_{\mathrm{e}}^{\prime}\left(\boldsymbol{\sigma}_{0}, \Delta \boldsymbol{\varepsilon}_{\mathrm{v}}\right) \Delta \boldsymbol{\varepsilon}^{\prime}=\overline{\mathbf{D}}_{\mathrm{e}}^{\prime} \Delta \boldsymbol{\varepsilon}^{\prime}
$$

In this equation, $\overline{\mathbf{D}}_{\mathrm{e}}^{\prime}$ is evaluated using the initial stress state $\boldsymbol{\sigma}_{0}^{\prime}$ and the total volumetric strain increment $\Delta \varepsilon_{\mathrm{v}}$. For the linear elasticity that occurs in conventional plasticity models, the trial stress increment $\Delta \boldsymbol{\sigma}_{\mathrm{e}}^{\prime}$ can be used later in the stress integration procedure. For non-linear elasticity, however, the elastic trial stress increment $\Delta \overline{\boldsymbol{\sigma}}_{\mathrm{e}}$ is used only to check if the stress state 


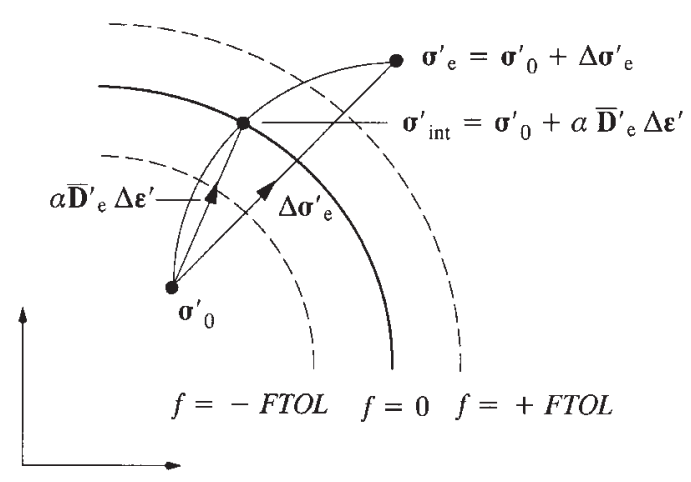

Figure 1. Yield surface intersection: elastic to plastic transition.

has changed from elastic to plastic. Such a change occurs if

$$
\begin{gathered}
f\left(\boldsymbol{\sigma}_{0}^{\prime}, u_{\mathrm{w} 0}, \kappa_{0}\right)<0 \\
f\left(\boldsymbol{\sigma}_{0}^{\prime}+\Delta \boldsymbol{\sigma}_{\mathrm{e}}^{\prime}, u_{\mathrm{w} 0}+\Delta u_{\mathrm{w}}, \kappa_{0}\right)=f\left(\boldsymbol{\sigma}_{\mathrm{e}}^{\prime}, u_{\mathrm{w}}, \kappa_{0}\right)>0
\end{gathered}
$$

and it is then necessary to ascertain the fraction of $\Delta \boldsymbol{\varepsilon}^{\prime}$ that moves the stresses from $\boldsymbol{\sigma}_{0}^{\prime}$ to the stress state $\boldsymbol{\sigma}_{\text {int }}^{\prime}$ on the yield surface. This situation, shown in Figure 1, may arise many times during the course of an elastoplastic finite-element analysis and needs to be handled efficiently and accurately. Note that in Figure 1 the exact yield condition $f\left(\boldsymbol{\sigma}^{\prime}, u_{\mathrm{w}}, \kappa\right)=0$ is replaced by the approximation $\left|f\left(\boldsymbol{\sigma}^{\prime}, u_{\mathrm{w}}, \kappa\right)\right| \leqslant$ FTOL, where FTOL is a small positive tolerance. This allows for the effects of finite precision arithmetic and modifies the transition condition to $f\left(\boldsymbol{\sigma}_{0}^{\prime}, u_{\mathrm{w} 0}, \kappa_{0}\right)$ $<-$ FTOL) and $f\left(\sigma_{\mathrm{e}}^{\prime}, u_{\mathrm{w}}, \kappa_{0}\right)>+$ FTOL. Suitable values for the yield surface tolerance are typically in the range $10^{-6}-10^{-9}$.

The problem of finding the stresses at the yield surface intersection point $\boldsymbol{\sigma}_{\text {int }}^{\prime}$ is equivalent to finding the scalar quantity $\alpha$ which satisfies the non-linear equation

$$
f\left(\boldsymbol{\sigma}_{0}^{\prime}+\alpha \overline{\mathbf{D}}_{\mathrm{e}}^{\prime} \Delta \boldsymbol{\varepsilon}^{\prime}, u_{\mathrm{w}}+\alpha \Delta u_{\mathrm{w}}, \kappa_{0}\right)=f\left(\boldsymbol{\sigma}_{\text {int }}^{\prime}, u_{\mathrm{w}}+\alpha \Delta u_{\mathrm{w}}, \kappa_{0}\right)=0
$$

Since the pore pressure $u_{\mathrm{w}}$ is treated as a strain component, the same fraction $\alpha$ should apply to both $\Delta \boldsymbol{\varepsilon}$ and $\Delta u_{\mathrm{w}}$. A value of $\alpha=0$ indicates that $\Delta \boldsymbol{\varepsilon}^{\prime}$ causes purely plastic deformation, while a value of $\alpha=1$ indicates purely 'elastic' deformation. Thus, for an elastic to plastic transition, we have $0<\alpha<1$ and the 'elastic' part of the stress increment is given by $\alpha \overline{\mathbf{D}}_{\mathrm{e}}^{\prime} \Delta \boldsymbol{\varepsilon}^{\prime}$. For the MCC model, the secant elastic stress-strain matrix $\overline{\mathbf{D}}_{\mathrm{e}}^{\prime}$ can be evaluated using the initial stress $\sigma_{0}$ and the strain increment $\alpha \Delta \varepsilon^{\prime}$. This means the trial elastic stress state, for a given initial stress and imposed strain increment, is exact and leads to accurate estimates of the intersection stress $\boldsymbol{\sigma}_{\text {int }}^{\prime}$.

Equation (25) defines a single non-linear equation in $\alpha$ and can be solved by a variety of numerical methods including bisection, regula-falsi, modified regula-falsi, secant, and NewtonRaphson. Sloan et al. [34] used the Pegasus algorithm to solve Equation (25). 


\section{Stress integration with substepping}

For given strain and pore pressure increments, $\Delta \boldsymbol{\varepsilon}$ and $\Delta u_{\mathrm{w}}$, the constitutive relations to be integrated at each Gauss point are described by Equations (11) and (12) as

$$
\begin{gathered}
\frac{\mathrm{d} \boldsymbol{\sigma}^{\prime}}{\mathrm{d} T}=\mathbf{D}_{\mathrm{ep}}^{\prime} \Delta \boldsymbol{\varepsilon}^{\prime} \\
\frac{\mathrm{d} \kappa}{\mathrm{d} T}=\mathbf{R}_{\mathrm{ep}}^{\prime} \Delta \boldsymbol{\varepsilon}^{\prime}
\end{gathered}
$$

where the pseudotime $T$ lies in the range

$$
0 \leqslant T \leqslant 1
$$

These equations describe a system of ordinary differential equations with initial conditions $\boldsymbol{\sigma}^{\prime}=\boldsymbol{\sigma}_{0}^{\prime}$ and $\kappa=\kappa_{0}$ at the start of the increment where $T=0$ and $t=t_{0}$. A wide variety of explicit methods may be used to integrate these equations and find the stresses and hardening parameter at the end of the increment, but we will focus on the approach first proposed by Sloan [23]. This strategy is attractive for finite-element analysis because it attempts to control the errors in the stresses and hardening parameter arising from the approximate integration of the constitutive law. The error control is implemented by using a local error measure to automatically subincrement the imposed strain increment $\Delta \boldsymbol{\varepsilon}$. For each subincrement, the local error is found by taking the difference between a second-order-accurate modified Euler solution and a first-order-accurate Euler solution. Once the local error has been computed for a given step, the size of the next step is determined using an expression for the dominant error term. This type of error control permits the size of each subincrement to vary throughout the integration process, depending on the non-linearity of the constitutive relations.

Consider a pseudo-time subincrement in the range $0<\Delta T_{n} \leqslant 1$ and let the subscripts $n-1$ and $n$ denote quantities evaluated at the pseudo-times $T_{n-1}$ and $T_{n}=T_{n-1}+\Delta T_{n}$. With the explicit Euler method, the values for $\boldsymbol{\sigma}^{\prime}$ and $\kappa$ at the end of a pseudo-time step $\Delta T_{n}$ are found from

$$
\begin{aligned}
& \boldsymbol{\sigma}_{n}^{\prime}=\boldsymbol{\sigma}_{n-1}^{\prime}+\Delta \boldsymbol{\sigma}_{1}^{\prime} \\
& \kappa_{n}=\kappa_{n-1}+\Delta \kappa_{1}
\end{aligned}
$$

where

$$
\begin{aligned}
\Delta \boldsymbol{\sigma}_{1}^{\prime} & =\mathbf{D}_{\mathrm{ep}}^{\prime}\left(\boldsymbol{\sigma}_{n-1}^{\prime},\left(u_{\mathrm{w}}\right)_{n-1}, \kappa_{n-1}\right) \Delta \boldsymbol{\varepsilon}_{n}^{\prime} \\
\Delta \kappa_{1} & =\mathbf{R}_{\mathrm{ep}}^{\prime}\left(\boldsymbol{\sigma}_{n-1}^{\prime},\left(u_{\mathrm{w}}\right)_{n-1}, \kappa_{n-1}\right) \Delta \boldsymbol{\varepsilon}_{n}^{\prime}
\end{aligned}
$$

and

$$
\Delta \varepsilon_{n}^{\prime}=\Delta T_{n} \Delta \varepsilon^{\prime}
$$

A more accurate estimate of the stresses and hardening parameter at the end of the interval $\Delta T_{n}$ can be found using the modified Euler procedure. This gives

$$
\begin{aligned}
& \hat{\boldsymbol{\sigma}}_{n}^{\prime}=\boldsymbol{\sigma}_{n-1}^{\prime}+\frac{1}{2}\left(\Delta \boldsymbol{\sigma}_{1}^{\prime}+\Delta \boldsymbol{\sigma}_{2}^{\prime}\right) \\
& \hat{\kappa}_{n}=\kappa_{n-1}+\frac{1}{2}\left(\Delta \kappa_{1}+\Delta \kappa_{2}\right)
\end{aligned}
$$


where $\Delta \boldsymbol{\sigma}_{1}^{\prime}$, and $\Delta \kappa_{1}$, are computed from the Euler scheme and

$$
\begin{aligned}
\Delta \boldsymbol{\sigma}_{2}^{\prime} & =\mathbf{D}_{\mathrm{ep}}^{\prime}\left(\boldsymbol{\sigma}_{n-1}^{\prime}+\Delta \boldsymbol{\sigma}_{1}^{\prime},\left(u_{\mathrm{w}}\right)_{n-1}+\Delta T \Delta u_{\mathrm{w}}, \kappa_{n-1}+\Delta \kappa_{1}\right) \Delta \boldsymbol{\varepsilon}_{n}^{\prime} \\
\Delta \kappa_{2} & =\mathbf{R}_{\mathrm{ep}}^{\prime}\left(\boldsymbol{\sigma}_{n-1}^{\prime}+\Delta \boldsymbol{\sigma}_{1}^{\prime},\left(u_{\mathrm{w}}\right)_{n-1}+\Delta T \Delta u_{\mathrm{w}}, \kappa_{n-1}+\Delta \kappa_{1}\right) \Delta \boldsymbol{\varepsilon}_{n}^{\prime}
\end{aligned}
$$

Note that elastoplastic matrices $\mathbf{D}_{\mathrm{ep}}^{\prime}$ and $\mathbf{R}_{\mathrm{ep}}^{\prime}$ are evaluated at the start of the subincrement for the first-order-accurate Euler updates (27) and at the end of the subincrement for the secondorder-accurate modified Euler updates (29). Since the local error in the Euler and modified Euler solutions is $O\left(\Delta T^{2}\right)$ and $O\left(\Delta T^{3}\right)$, respectively, the error in $\boldsymbol{\sigma}_{n}$ and $\kappa_{n}$ can be estimated from

$$
\left\{\begin{array}{c}
\hat{\boldsymbol{\sigma}}_{n}^{\prime} \\
\hat{\kappa}_{n}
\end{array}\right\}-\left\{\begin{array}{c}
\boldsymbol{\sigma}_{n}^{\prime} \\
\kappa_{n}
\end{array}\right\}=\left\{\begin{array}{l}
\frac{1}{2}\left(\Delta \boldsymbol{\sigma}_{2}^{\prime}-\Delta \boldsymbol{\sigma}_{1}^{\prime}\right) \\
\frac{1}{2}\left(\Delta \kappa_{2}-\Delta \kappa_{1}\right)
\end{array}\right\}
$$

Using any convenient norm, this quantity can be used to compute the relative error measure

$$
R_{n}=\frac{1}{2} \max \left\{\frac{\left\|\Delta \boldsymbol{\sigma}_{2}^{\prime}-\Delta \boldsymbol{\sigma}_{1}^{\prime}\right\|}{\left\|\hat{\boldsymbol{\sigma}}_{n}^{\prime}\right\|}, \frac{\left|\Delta \kappa_{2}-\Delta \kappa_{1}\right|}{\hat{\kappa}_{n}}\right\}
$$

where the stresses are treated separately from the hardening parameter to allow for differences of scale. Once this error measure has been computed, the current strain subincrement is accepted if $R_{n}$ is not greater than some prescribed tolerance, STOL, and rejected otherwise. Regardless of whether the subincrement is accepted or rejected, the next pseudo-time step is found from the relation

$$
\Delta T_{n+1}=q \Delta T_{n}
$$

where $q$ is chosen so that $R_{n+1}$ satisfies the constraint

$$
R_{n+1} \leqslant \mathrm{STOL}
$$

Since the local truncation error in the Euler method is $O\left(\Delta T^{2}\right)$, it follows from (31) that

$$
R_{n+1} \approx q^{2} R_{n}
$$

which, combined with constraint (32), gives

$$
q \leqslant \sqrt{\mathrm{STOL} / R_{n}}
$$

This procedure for determining $q$ is based on the expression for the dominant error term. Because this approximation may become inaccurate for strongly non-linear behaviour, it is prudent to choose $q$ conservatively to minimize the number of rejected strain subincrements. Numerical experiments on a wide variety of plasticity problems suggest that a suitable strategy for computing $q$ is to set

$$
q=0.9 \sqrt{\mathrm{STOL} / R_{n}}
$$

and also constrain it to lie within the limits

$$
0.1 \leqslant q \leqslant 1.1
$$

so that

$$
0.1 \Delta T_{n-1} \leqslant \Delta T_{n} \leqslant 1.1 \Delta T_{n-1}
$$

In the above, the coefficient of 0.9 acts as a safety factor, since it aims to prevent the step control mechanism from choosing strain subincrements which just fail to meet the local error tolerance. Restricting the growth of consecutive strain subincrements to $10 \%$ serves the same purpose. 
Numerical trials suggest that raising the limit on the growth factor for consecutive subincrements to $100 \%$ has only a marginal influence on the performance of the algorithm. Although relaxing these various constraints leads to larger subincrement sizes and fewer strain subincrements overall, this benefit is counteracted by an increased number of failed subincrements. Two final controls, of lesser importance than the above refinements, impose a minimum step size, $\Delta T_{\min }$, and prohibit the step size from growing immediately after a failed subincrement. The first condition adds robustness and is rarely invoked unless the constitutive law has gradient singularities. The second condition forces the two strain subincrements following a failure to be of the same size, and is useful for negotiating stress-strain paths with sharp changes in curvature.

The integration scheme is started by applying (26) and (27) with the known strains $\Delta \boldsymbol{\varepsilon}^{\prime}$, the initial stresses $\sigma_{0}$, the initial hardening parameter $\kappa_{0}$, and an initial pseudo-time step $\Delta T_{1}$. To allow for the case where no substeps are needed, the first trial substep $\Delta T_{1}$, is set to unity. If the relative error, defined by Equation (30), does not exceed the specified tolerance STOL, then the current subincrement is accepted and the stresses and hardening parameter are updated using either (26) or (28). In practice, it is advantageous to employ the higher-order update rather than the lower-order update, since this is the most accurate of the two and has already been calculated. The extra accuracy of the higher-order update compensates for the fact that (30) is only a local and not a global error indicator. Indeed it can be shown that, provided the constitutive laws are sufficiently smooth, this approach gives a global integration error which is directly proportional to STOL [38]. After a successful subincrement, the new stresses and hardening parameter are restored to the yield surface using the procedure described in the previous section. If $R_{n}>\mathrm{STOL}$, the solution is rejected and a smaller step size is computed using Equations (33) and (34). The stage is then repeated and, if necessary, the step size is reduced further until a successful subincrement size is obtained. Regardless of whether the current subincrement is a success or a failure, the size of the next strain subincrement is found using (33) and (34). The subincrements may become larger or smaller in successive steps, depending on the error that is calculated from Equation (30). The end of the integration procedure is reached when the entire increment of strain is applied so that

$$
\sum \Delta T=T=1
$$

The complete explicit modified Euler algorithm for unsaturated soil models, which includes all of the refinements described in the previous sections, may be summarized as follows.

\section{Explicit modified Euler algorithm for unsaturated soils.}

1. Enter with initial stresses $\boldsymbol{\sigma}_{0}^{\prime}$, initial pore water pressure $u_{\mathrm{w} 0}$, initial hardening parameter $\kappa_{0}$, the strain increment for the current step $\Delta \varepsilon$, the pore pressure increment $\Delta u_{\mathrm{w}}$, and the error tolerance for the stresses STOL.

2. Compute the stress increment $\Delta \boldsymbol{\sigma}_{\mathrm{e}}^{\prime}$ and the trial elastic stress state $\boldsymbol{\sigma}_{\mathrm{e}}^{\prime}$ according to

$$
\begin{gathered}
\Delta \boldsymbol{\sigma}_{\mathrm{e}}^{\prime}=\overline{\mathbf{D}}_{\mathrm{e}}^{\prime}\left(\boldsymbol{\sigma}_{0}^{\prime}, \Delta \boldsymbol{\varepsilon}_{\mathrm{v}}\right) \Delta \boldsymbol{\varepsilon}^{\prime} \\
\boldsymbol{\sigma}_{\mathrm{e}}^{\prime}=\boldsymbol{\sigma}_{0}^{\prime}+\Delta \boldsymbol{\sigma}_{\mathrm{e}}^{\prime}
\end{gathered}
$$

If $f\left(\boldsymbol{\sigma}_{\mathrm{e}}^{\prime}, u_{\mathrm{w} 0}+\Delta u_{\mathrm{w}}, \kappa_{0}\right) \leqslant \mathrm{FTOL}$ then the stress increment is purely elastic, so set $\boldsymbol{\sigma}_{1}^{\prime}=\boldsymbol{\sigma}_{\mathrm{e}}^{\prime}$ and $\kappa_{1}=\kappa_{0}$ and go to step 16 . 
3. If $f\left(\boldsymbol{\sigma}_{0}^{\prime}, u_{\mathrm{w} 0}, \kappa_{0}\right)<-$ FTOL and $f\left(\boldsymbol{\sigma}_{\mathrm{e}}^{\prime}, u_{\mathrm{w} 0}+\Delta u_{\mathrm{w}}, \kappa_{0}\right)>$ FTOL then the stress point undergoes a transition from elastic to plastic behaviour. Compute the portion of $\Delta \boldsymbol{\varepsilon}^{\prime}$ that corresponds to purely elastic deformation, $\alpha$, using the intersection scheme presented earlier and go to step 5 .

4. If $\left|f\left(\boldsymbol{\sigma}_{0}^{\prime}, u_{\mathrm{w} 0}, \kappa_{0}\right)\right| \leqslant$ FTOL and $f\left(\boldsymbol{\sigma}_{\mathrm{e}}^{\prime}, u_{\mathrm{w} 0}+\Delta u_{\mathrm{w}}, \kappa_{0}\right)>$ FTOL, the stress increment is purely plastic, so set $\alpha=0$.

5. Update the stresses at the onset of plastic yielding as $\boldsymbol{\sigma}_{0}^{\prime} \leftarrow \boldsymbol{\sigma}_{0}^{\prime}+\alpha \overline{\mathbf{D}}_{\mathrm{e}}^{\prime}\left(\boldsymbol{\sigma}_{0}^{\prime}, \alpha \Delta \varepsilon_{\mathrm{v}}\right) \Delta \boldsymbol{\varepsilon}^{\prime}$. Then compute the portion of $\Delta \boldsymbol{\varepsilon}^{\prime}$ that corresponds to plastic deformation according to $\Delta \boldsymbol{\varepsilon}^{\prime} \leftarrow(1-\alpha) \Delta \boldsymbol{\varepsilon}^{\prime}$.

6. Set $T=0$ and $\Delta T=1$.

7. While $T<1$, do steps $8-15$.

8. Compute $\Delta \boldsymbol{\sigma}_{i}^{\prime}$ and $\Delta \kappa_{i}$ for $i=1-2$ using

$$
\begin{gathered}
\Delta \boldsymbol{\sigma}_{\mathrm{e} i}^{\prime}=\mathbf{D}_{\mathrm{e} i} \Delta T \Delta \boldsymbol{\varepsilon} \\
\Delta \boldsymbol{\sigma}_{i}^{\prime}=\Delta \boldsymbol{\sigma}_{\mathrm{e} i}^{\prime}-\Delta \lambda_{i} \mathbf{D}_{\mathrm{e} i} \mathbf{a}_{g i} \\
\Delta \kappa_{i}=\Delta \lambda_{i} B_{i}
\end{gathered}
$$

where

$$
\begin{gathered}
\mathbf{D}_{\mathrm{e} i}=\mathbf{D}_{\mathrm{e}}\left(\boldsymbol{\sigma}_{i}^{\prime}\right) \\
\Delta \lambda_{i}=\max \left\{\frac{\mathbf{a}_{f j}^{\mathrm{T}} \Delta \boldsymbol{\sigma}_{\mathrm{e} i}^{\prime}+C_{i} \Delta T \Delta u_{\mathrm{w}}}{A_{i}+\mathbf{a}_{f i}^{\mathrm{T}} \mathbf{D}_{\mathrm{e} i} \mathbf{a}_{g i}}, 0\right\} \\
\mathbf{a}_{g i}=\left(\frac{\partial g}{\partial \boldsymbol{\sigma}^{\prime}}\right)_{i} \\
\mathbf{a}_{f i}=\left(\frac{\partial f}{\partial \boldsymbol{\sigma}^{\prime}}\right)_{i} \\
B_{i}=\frac{\partial p_{\mathrm{c}}}{\partial \varepsilon_{\mathrm{v}}^{\mathrm{p}}}\left(\frac{\partial g}{\partial p^{\prime}}\right)_{i} \\
A_{i}=-(\partial f / \partial \kappa) B_{i} \\
C_{i}=\left(\frac{\partial f}{\partial u_{\mathrm{w}}}\right)_{i}
\end{gathered}
$$

are evaluated at $\left(\tilde{\boldsymbol{\sigma}}_{i}^{\prime},\left(u_{\mathrm{w}}\right)_{i}, \tilde{\kappa}_{i}\right)$, and

$$
\begin{array}{lll}
\tilde{\boldsymbol{\sigma}}_{1}^{\prime}=\boldsymbol{\sigma}_{T}^{\prime}, & \tilde{\kappa}_{1}=\kappa_{T}, & \left(u_{\mathrm{w}}\right)_{1}=u_{\mathrm{w} 0}+T \Delta \mathrm{u}_{\mathrm{w}} \\
\tilde{\boldsymbol{\sigma}}_{2}^{\prime}=\boldsymbol{\sigma}_{T}^{\prime}+\Delta \boldsymbol{\sigma}_{1}, & \tilde{\kappa}_{2}=\kappa_{T}+\Delta \kappa_{1}, & \left(u_{\mathrm{w}}\right)_{2}=u_{\mathrm{w} 0}+(T+\Delta T) \Delta u_{\mathrm{w}}
\end{array}
$$

9. Compute the new stresses and hardening parameter and hold them in temporary storage according to

$$
\begin{aligned}
& \tilde{\boldsymbol{\sigma}}_{T+\Delta T}^{\prime}=\boldsymbol{\sigma}_{T}^{\prime}+\frac{1}{2}\left(\Delta \boldsymbol{\sigma}_{1}^{\prime}+\Delta \boldsymbol{\sigma}_{2}^{\prime}\right) \\
& \tilde{\kappa}_{T+\Delta T}=\kappa_{T}+\frac{1}{2}\left(\Delta \kappa_{1}+\Delta \kappa_{2}\right)
\end{aligned}
$$


10. Determine the relative error for the current substep from

$$
R_{T+\Delta T}=\max \left\{\frac{\left\|\Delta \boldsymbol{\sigma}_{2}^{\prime}-\Delta \boldsymbol{\sigma}_{1}^{\prime}\right\|}{2\left\|\tilde{\boldsymbol{\sigma}}_{T+\Delta T}^{\prime}\right\|}, \frac{\left|\Delta \kappa_{2}-\Delta \kappa_{1}\right|}{2 \tilde{\kappa}_{T+\Delta T}}, \text { EPS }\right\}
$$

where EPS is a machine constant indicating the smallest relative error that can be calculated.

11. If $R_{T+\Delta T}>$ STOL the substep has failed and a smaller pseudo-time step needs to be found by extrapolation. First compute

$$
q=\max \left\{0.9 \sqrt{\mathrm{STOL} / R_{T+\Delta T}}, 0.1\right\}
$$

and then set

$$
\Delta T \leftarrow \max \left\{q \Delta T, \Delta T_{\min }\right\}
$$

before returning to step 8 .

12. The substep is successful, so update the stresses and the hardening parameter according to

$$
\begin{aligned}
& \boldsymbol{\sigma}_{T+\Delta T}^{\prime}=\tilde{\boldsymbol{\sigma}}_{T+\Delta T}^{\prime} \\
& \kappa_{T+\Delta T}=\tilde{\kappa}_{T+\Delta T}
\end{aligned}
$$

13. If $\left|f\left(\boldsymbol{\sigma}_{T+\Delta T}^{\prime}, u_{\mathrm{w} 0}+(T+\Delta T) \Delta u_{\mathrm{w}}, \kappa_{T+\Delta T}\right)\right|>$ FTOL, then correct $\boldsymbol{\sigma}_{T+\Delta T}^{\prime}$ and $\kappa_{T+\Delta T}$ back to the yield surface using the algorithm presented [34].

14. Extrapolate to obtain the size of the next substep by computing

$$
q=\min \left\{0.9 \sqrt{\mathrm{STOL} / R_{T+\Delta T}}, 1.1\right\}
$$

If the previous step failed, limit the step size growth further by enforcing

$$
q=\min \{q, 1\}
$$

Update pseudo-time and compute new step size according to

$$
\begin{gathered}
T \leftarrow T+\Delta T \\
\Delta T \leftarrow q \Delta T
\end{gathered}
$$

15. Ensure the next step size is not smaller than the minimum step size and check that integration does not proceed beyond $T=1$ by setting

$$
\Delta T \leftarrow \max \left\{\Delta T, \Delta T_{\min }\right\}
$$

and then

$$
\Delta T \leftarrow \min \{\Delta T, 1-T\}
$$

16. Exit with stresses $\boldsymbol{\sigma}_{1}$, and hardening parameter $\kappa_{1}$, at end of increment with $T=1$.

The stress integration algorithm presented above is a generalization of the scheme of Sloan et al. [39] to incorporate the pore pressure in the yield function. As this variable is treated as an additional strain component, it is assumed that it may be subincremented at the same rate as the other strain components. This provides a consistent means for determining what pore pressure values should be used when computing any pore pressure-dependent quantities, such as the yield function and its gradients. The algorithm dealing with elastoplastic unloading, which is detailed 
in Reference [39] for conventional soil models, is not included here because the modified subincrementation technique should capture this special case.

\section{CONCLUSIONS}

A finite-element formulation for saturated and unsaturated soils is presented. This formulation is simple to implement, employs algorithms which are similar to those for saturated soils, and treats the suction as a strain variable (instead of a stress variable) in the constitutive relations.

An adaptive time-stepping scheme is proposed for solving the discretized global equations that govern the deformation and fluid flow in saturated-unsaturated soils. This scheme automatically adjusts the time-step size so that the temporal integration error in the displacements lies close to a specified tolerance, thus removing the need to determine a suitable time-step regime by trial and error. In formulating the algorithm for unsaturated soils, special care is given to the system non-linearity caused by suction-dependent plastic yielding, a suctiondependent degree of saturation and saturation-dependent permeability.

An explicit stress integration scheme is proposed for solving the constitutive equations with the suction treated as a additional strain component. This scheme is based upon the modified Euler method with automatic subincrementation and error control $[24,39]$. In generalizing this scheme to cover unsaturated soil behaviour, special attention is again given to suctiondependent plastic yielding. When applying subincrementation, the same rate is assumed for all strain components, including the suction. This assumption is consistent with the fact that the suction and the strains (displacements) are solved from the global equations simultaneously. Algorithms for computing the elastic trial stresses, the intersection with the yield surface, and the yield surface drift correction are described in detail, together with the explicit stress integration procedure.

\section{REFERENCES}

1. Richards BG. Modelling interactive load-deformation and flow processes in soils, including unsaturated and swelling soils. John Jaeger Memorial Lecture, Proceedings of the 6th Asut-NZ Conference on Geomechanics, Christchurch, New Zealand, 1992; 18-37.

2. Li J, Cameron DA. Finite element analysis of deep beams in expansive clays. In: Alonso EE, Delage P (eds). Proceedings of the 1st International Conference on Unsaturated Soils, vol. 2. Balkema: Rotterdam, 1995; 1109-1115.

3. Alonso EE, Batlle F, Gens A, Lloret A. Consolidation analysis of partially saturated soils. Proceedings of the 6th International Conference on Numerical Methods in Geomechanics, vol. 2. Innsbruck, 1988; 1303-1308.

4. Thomas HR, He Y. Analysis of coupled heat, moisture and air transfer in a deformable unsaturated soil. Geotechnique 1995; 45:677-689.

5. Loret B, Khalili N. A three-phase model for unsaturated soils. International Journal for Numerical and Analytical Methods in Geomechanics 2000; 24:893-927.

6. Li X, Zienkiewicz OC. Multiphase flow in deforming porous media and finite element solutions. Computers and Structures 1992; 45:211-227.

7. Olivella S, Carrera J, Gens A, Alonso EE. Non-isothermal multiphase flow pf brine and gas through saline media. Transport in Porous Media 1994; 15:271-293.

8. Gatmiri B, Tavakoli S, Moussavi J, Delage P. Numerical approach of elastoplastic consolidation of unsaturated soils. In: Alonso EE, Delage P (eds). Proceedings of the 1st International Conference on Unsaturated Soil, vol. 2. Balkema: Rotterdam, 1995; 1057-1064.

9. Thomas HR, King SD. Coupled temperature/capillary potential variations in unsaturated soil. Journal of Engineering Mechanics ASCE 1991; 117(11):2475-2491.

10. Wood WL. Control of Crank-Nicholson noise in the numerical solution of the heat conduction equation. International Journal for Numerical Methods in Engineering 1977; 11:1059-1065.

11. Alonso EE, Gens A, Josa A. A constitutive model for partially saturated soils. Geotechnique 1990; 40:405-430.

Copyright (C) 2003 John Wiley \& Sons, Ltd.

Int. J. Numer. Anal. Meth. Geomech. 2003; 27:745-765 
12. Roscoe KH, Burland JB. On the generalised stress-strain behaviour of "wet" clay. Engineering Plasticity. Cambridge University Press: Cambridge, MA, 1968; 535-609.

13. Schrefler BA, Zhan XY. A fully coupled model for water flow and airflow in deformable porous media. Water Resources Research 1993; 29:155-167.

14. Cui YJ, Delage P, Sultan N. An elastoplastic model for compacted soils. In: Alonso EE, Delage P (eds). Proceedings of the 1st International Conference on Unsaturated Soil, vol. 2. Balkema: Rotterdam, 1995; 703-709.

15. Bolzon G, Schrefler BA, Zienkiewicz OC. Elastoplastic soil constitutive laws generalised to partially saturated states. Geotechnique 1996; 46:279-289.

16. Wheeler SJ, Sivakumar V. An elastoplastic critical state framework for unsaturated soil. Geotechnique 1995; 45: $35-53$.

17. Kohgo Y, Nakano M, Miyazaki T. Theoretical aspects of constitutive modelling for unsaturated soils. Soils and Foundations 1993; 33:49-63.

18. Modaressi A, Abou-Bekr N. A unified approach to model the behaviour of saturated and unsaturated soils. Proceedings of the 8th International Conference on Computer Methods and Advances in Geomechanics. Balkema: Rotterdam, 1994; 1507-1513.

19. Gens A. Constitutive modelling: application to compacted soils. In: Alonso EE, Delage P (eds). Proceedings of 1st International Conference on Unsaturated Soil, vol. 3. Balkema: Rotterdam, 1995; 1179-1200.

20. Geiser F. Applicability of a general effective stress concept to unsaturated soils. In: Rahardjo H, et al. (eds). Unsaturated Soils for Asia, Balkema: Rotterdam, 2000; 101-105.

21. Khalili N. Application of the effective stress principle to volume change in unsaturated soils. In: Rahardjo $\mathrm{H}$, et al. (eds). Unsaturated Soils for Asia, Balkema: Rotterdam, 2000; 119-124.

22. Wissmann JW, Hauck C. Efficient elasto-plastic finite element analysis with higher order stress point algorithms. Computers and Structures 1983; 17:89-95.

23. Sloan SW. Substepping schemes for the numerical integration of elastoplastic stress-strain relations. International Journal for Numerical Methods in Engineering 1987; 24:893-911.

24. Vaunat J, Cante JC, Ledesma A, Gens A. A stress point algorithm for an elastoplastic model in unsaturated soils. International Journal of Plasticity 2000; 16:121-141.

25. Runesson K. Implicit integration of elastoplastic relations with reference to soils. International Journal for Numerical and Analytical Methods in Geomechanics 1987; 11:315-321.

26. Zhang HW, Heeres OM, de Borst R, Schrefler BA. Implicit integration of a generalized plasticity constitutive model for partially saturated soil. Engineering Computations 2001; 18:314-336.

27. Simo JC, Taylor RL. A return mapping algorithm for plane stress elasto-plasticity. International Journal for Numerical Methods in Engineering 1986; 22:647-760.

28. Van Genuchten MT. A closed form equation of predicting the hydraulic conductivity of unsaturated soils. Soil Science of America Journal 1980; 44:892-898.

29. Hillel D. Soil and Water-Physical Principles and Processes. Academic Press: New York, 1971.

30. Booker JR, Small JC. An investigation of the stability of numerical solutions of Biot's equations of consolidation. International Journal of Solids and Structures 1975; 11:907-917.

31. Vermeer PA, Verruijt A. An accuracy condition for consolidation by finite elements. International Journal for Numerical and Analytical Methods in Geomechanics 1981; 5:1-14.

32. Wood LW. Practical Time-stepping Schemes, Clarendon Press, Oxford, 1990.

33. Sloan SW, Abbo AJ. Biot consolidation analysis with automatic time stepping and error control, Part 1: Theory and Implementation. International Journal for Numerical and Analytical Methods in Geomechanics 1999; 23:467-492.

34. Sloan SW, Abbo AJ. Biot consolidation analysis with automatic time stepping and error control, Part 2: Application. International Journal for Numerical and Analytical Methods in Geomechanics 1999; 23:493-529.

35. Thomas RM, Gladwell I. Variable-order variable step algorithms for second-order systems, Part 1: The methods. International Journal for Numerical Methods in Engineering 1988; 26:39-53.

36. Sheng D, Sloan SW, Yu HS. Aspects of finite element implementation of critical state models. Computational Mechanics 2000; 26(2):185-196.

37. Sloan SW, Abbo AJ, Sheng D. Refined explicit integration of elasto-plastic models with automatic error control. Engineering Computations 2001; 18:121-154.

38. Shampine LF. Numerical Solution of Ordinary Differential Equations. Chapman \& Hall: London, 1994.

39. Sloan SW, Sheng D, Abbo AJ. Accelerated initial stiffness schemes for elastoplasticity. International Journal for Numerical and Analytical Methods in Geomechanics 2000; 24:579-599. 